\title{
Cuestiones para el debate entre economía y arte. Reflexiones desde el contexto cubano
}

Questions for Debate between Economics and Art. Reflections from Cuban Context

\section{Jorge Alfredo Carballo Concepción \\ Universidad de La Habana jorge.carballo@flacso.uh.cu}

Resumen: La relación entre arte y capital es compleja. Las transformaciones de la Economía Mundial que han tenido lugar desde finales del siglo xIx hasta la actualidad, son el resultado del desarrollo acelerado de las fuerzas productivas en un entorno de máxima competencia. Es por ello que el contexto actual impone desafíos a los artistas, los emprendedores y las instituciones culturales. Los creadores encaran su realización profesional desde situaciones concretas desiguales, mediadas por las relaciones mercantiles, la necesidad de fuentes de financiación, los avances tecnológicos, las redes sociales, etc. Asimismo, intentan convertir en bienes y servicios su creatividad y talento artístico, pero a su vez, necesitan encontrar un balance entre el componente creativo, funcional y el componente productivo, para poder alcanzar los objetivos y metas propuestas, a través de la gestión innovadora, con posibilidades de escala. Desde esta lógica, vale la pena situar 
en contexto el lugar de los debates sobre Economía y Arte en las transformaciones socioeconómicas que tienen lugar en Cuba, de forma que se promueva el debate profundo, y se avance en el entendimiento de sus determinantes socioeconómicos.

Palabras clave: cultura, arte, economía, mercado, producción, fuerza de trabajo.

Abstract: The relationship between art and capital is complex. The transformations of the world economy that have taken place since the end of the $19^{\text {th }}$ century to the present day are the result of the accelerated development of productive forces in an environment of maximum competition. This is why the current context imposes challenges on artists, entrepreneurs and cultural institutions. Creators face their professional realization from unequal concrete situations, mediated by mercantile relations, the need for sources of financing, technological advances, social networks, etc. Likewise, they try to turn their creativity and artistic talent into goods and services, but at the same time, they need to find a balance between the creative, functional and productive components, in order to achieve the proposed objectives and goals, through innovative management, with possibilities of scale. From these logics, it is worthwhile to place in context the place of the debates on Economy and Art in the socioeconomic transformations taking place in Cuba, so as to promote a deep debate, and to advance in the understanding of their socioeconomic determinants.

Keywords: culture, art, economics, market, production, workers.

Fecha de recepción: 8/4/2021

Fecha de aceptación: 9/8/2021 
Las Ciencias Económicas han intentado, tomando en consideración diversos postulados doctrinales, explicar y resolver asuntos concernientes al lugar de la cultura y la producción de arte en el conjunto de la economía, y se ha propuesto aplicar a aquellas, su vasto instrumental teórico y metodológico.

Mas esa aplicación ha sido realizada desde conceptualizaciones que responden a la dinámica de valorización en función del capital, y se centra en los patrones de consumo para conseguir que la esfera cultural aparezca sujeta a modelos homogeneizantes de oferta y demanda, sin tener en consideración las determinantes socioeconómicas del proceso de producción de arte.

Lejos está la época donde la cultura era asociada exclusivamente a las bellas artes, al ocio y a una formación intelectual más exigente. El avance incontenible de las ciencias sociales, signado por el cambio en el patrón de acumulación, nos conduce a múltiples definiciones que asocian la cultura y la producción de arte con la vida misma de las personas, expresada en costumbres, ideas y valores, como forma de expresión genuina del conjunto de toda la sociedad.

Es de interés para estudiosos del tema, emprendedores y artistas, avanzar en el entendimiento del lugar de la producción de arte dentro del sistema de relaciones sociales de producción donde se desempeñan. En este sentido, este trabajo es un pequeño aporte a los debates sobre esta problemática.

Desde una perspectiva histórica se puede decir que, en sus orígenes, la ciencia económica mostró escaso interés por profundizar los estudios de los bienes y servicios culturales. Se con- 
sideraba que la demanda de estos bienes era una dispendiosa extravagancia de la aristocracia o una peligrosa distracción de la clase trabajadora ${ }^{1}$.

La revolución marginalista de finales del siglo XIX tuvo también su impronta en los estudios y acercamientos de la ciencia económica a la cultura, como consecuencia de las transformaciones en el patrón de acumulación capitalista y el subsiguiente surgimiento del imperialismo como fase superior del modo de producción capitalista.

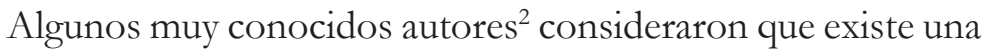
identificación entre el ámbito de la cultura y la economía, a partir de necesidades no cubiertas, disponibilidad de recursos y posibilidades de utilización de instrumentos de política económica. Sin embargo, no logran esclarecer la naturaleza de esas relaciones, ni se preocupan por estudiar las formas organizativas de la producción y tampoco tienen presente en su análisis las relaciones sociales de producción, en las cuales se enmarcan.

Karl Marx (1818-1883) puso atención en el análisis de las cuestiones culturales, específicamente en su relación con el resto de la estructura social. En su análisis, la cultura es entendida como el producto de las relaciones de producción, como un fenómeno que no está desligado del modo de producción de una sociedad. Asimismo, la considera como uno de los medios por los cuales se reproducen las relaciones sociales de producción, que permiten la permanencia en el tiempo de las condiciones de desigualdad entre las clases ${ }^{3}$.

1 Ver: SмITH, A. (1776): «Investigación de la naturaleza y causas de la riqueza de las naciones». Valladolid: Consejería de Educación y Cultura de la Junta de Castilla y León, Valladolid, 1996, pp. 425-426; Ricardo, D. (1821): «Principios de economía política y tributación». México: FCE, 1959 , p. 20.

2 Alfred Marshall (1842-1924), John Maynard Keynes (1883-1946) y Lionel Robbins (1898-1984). 3 Marx, C. y Engels, F. «El Manifiesto Comunista». Publicado en marzo de 1848 en Londres. La Habana: Editorial de Ciencias Sociales, 1979, pp. 30-32. 
Refiriéndose a esta temática expresó:

Respecto a las esferas de la ideología que se elevan por encima del aire, la religión, la filosofía, etc., todas tienen una existencia prehistórica, se pueden encontrar vestigios de su existencia en todo período histórico... La historia de la ciencia es la historia del intento de superar estos disparates o su sustitución por algo nuevo y menos absurdo. Las personas que se ocupan de esta tarea, pertenecen a su vez a esferas especiales de la división del trabajo y se presentan para trabajar en un terreno independiente. Y forman un grupo independiente dentro de la división social del trabajo; realizan sus predicciones, incluso sus errores, e influyen en el desarrollo de la sociedad, incluso en el desarrollo económico. Aunque al mismo tiempo, están bajo la influencia dominante del desarrollo económico. ${ }^{4}$

Antonio Gramsci (1891-1937) reconocía la importancia de los factores estructurales, especialmente la economía, y considerando las condiciones histórico concretas en que vivió, desarrolló creadoramente un concepto central: la hegemonía cultural ${ }^{5}$, donde los elementos fundamentales de la dominación capitalista no son los económicos, sino los de orden cultural. Para él, «el ingrediente esencial de la filosofía de la praxis más moderna - el vínculo entre pensamiento y acción_-, es el concepto histórico-filosófico de hegemonía». Gramsci define la hegemonía como el liderazgo cultural ejercido por la clase dirigente ${ }^{6}$.

El nacimiento de la Economía de la Cultura como área del conocimiento en las ciencias económicas modernas se puede fe-

4 Marx, C. y Engels, F. Selected Correspondence, pp. 482-483.

5 El concepto de hegemonía deriva del griego eghestai, que significa «conducir», «ser guía», «ser jefe», y del verbo eghemoneno, que quiere decir «ser guía», «proceden», «conducir», lo que da por derivación, «ser jefe», «mandar», «dominan». Con hegemonía, el griego antiguo designaba el mando supremo de los ejércitos. Se trata, pues, de un término militar.

6 GramscI, A. «Socialismo y Cultura», en Il Grido di Popolo, 1916, artículo reproducido en Ontología. Madrid: Sacristán de Siglo XXI, pp. 14-17. 
char con exactitud. Comenzó con la obra de Baumol y Bowen publicado en 1976 titulado Performing Arts-The Economic Dilemma o el «El dilema económico de las artes escénicas»? El dilema aparece debido a que un creciente nivel de bienestar económico o ingreso per cápita, que lleva a un aumento de la demanda de representaciones, conlleva un mayor coste de las representaciones escénicas.

A partir de este criterio, conceptos como costo, presupuesto, estrategia de mercado, demanda y oferta cultural, o financiación se fusionan con los elementos conceptuales tradicionales utilizados en el mundo del arte: creatividad, innovación, producción, o experiencia estética, para introducir métodos de análisis económicos donde se pone énfasis en la caracterización de los bienes y servicios culturales, cuyo análisis se creía ajeno al mercado.

De esta forma, se puede afirmar que el enfoque económico utilizado para el análisis de los aspectos de la relación entre Economía y Cultura, es el de la elección racional ${ }^{\beta}$, el cual hace distinción de las preferencias, las restricciones, los ingresos, los precios y el tiempo disponible y se convierte en referente teórico para el análisis económico de la cultura.

No hay dudas que los factores que influyen en la creación artística y el consumo cultural sean especialmente numerosos y que la relación entre ellos sea, por su esencia, más compleja que en el caso, digamos, de la producción y el consumo de

\footnotetext{
7 Baumol, W. y Bowen, W. (1966). Performing Arts. The Economic Dilemma. Cambridge, Mass: New York Twentieth Century Found, pp. 75-83.

8 Los resultados conseguidos gracias a este enfoque se han visto reconocidos por la concesión de varios premios Nobel de Economía, especialmente el conseguido por Gary Becker en 1992. El mismo enfoque de la elección racional ha sido utilizado por otros ganadores de ese premio, como Kenneth Arrow en 1972, Herbert Simon en 1978, Theodore Schultz en 1971, James Buchanan en 1986, Ronald Coase en 1991, Douglas North y Robert Fogel en 1993 y AmartyaSen en 1998.
} 
otro bien no cultural, pero el arte y la cultura están, en principio, sujetos a la escasez es decir, no son bienes libres; proporcionan utilidad a los individuos que las demandan, y necesitan recursos para su creación.

En definitiva, se ha buscado desmenuzar las características que hacen del arte y la actividad artística un bien sujeto a interés público; es decir, se trata de unos bienes y servicios que producen efectos externos positivos, cuyos beneficios no se agotan en las personas que los demandan y ofrecen como bien privado a través de los mecanismos de mercado.

Se comprende entonces que con este enfoque se excluyen del análisis las relaciones sociales de producción y su relación con el grado de desarrollo de las fuerzas productivas. Es imposible establecer con esta lógica, cual es el papel de los productores de arte dentro del sistema social, más allá de su posición con base en su éxito relativo.

\section{La producción de arte y el sistema económico mundial}

Desde el principio el arte tuvo un carácter social. Las maravillosas pinturas rupestres de Francia y España, fueron pintadas en las zonas más profundas e inaccesibles de las cuevas. No era una simple decoración, formaban parte de un ritual destinado a fines muy prácticos, conseguir el control de los bisontes, ciervos y caballos salvajes cazados para comer. La danza y las canciones tenían el mismo propósito.

Tras milenios solo ocasionalmente en contacto con el mercado, donde se realiza y se renueva con criterios mercantiles y comerciales, la producción de arte se ha convertido en objeto económico de aquel, gracias al poder que ejercen las nuevas tecnologías de la comunicación sobre nuestra vida, y que se nos presenta como un simple hecho comercial basado en la venta de 
experiencias personales y cuya esencia es la privatización de esos bienes y servicios culturales.

La industria cultural -un término de los años treinta creado por los sociólogos alemanes Theodor Adorno y Max Horkheimer - es uno de los sectores que está creciendo más rápido en la economía mundial. El cine, la radio, la televisión, la industria de la grabación, el turismo global, los centros destinados al entretenimiento, ciudades creativas, ciudades musicales, la moda, entre otros conforman la vanguardia comercial de la era del capitalismo cultural.

Estamos entonces en un escenario de capitalismo cultural en pleno auge y desarrollo, apropiándose no solo de los significados de la vida cultural y de las formas de comunicación artísticas que los interpretan, sino también de sus experiencias de vida. El arte, antaño adversario de los valores mercantiles, es ahora su primer apóstol y principal comunicador. Al fin, los fabricantes de experiencia constituyen un sector clave, quizás de los principales de la economía, y somos la primera cultura en la historia que emplee alta tecnología para manufacturar el más perdurable de los productos: la experiencia humana.

El arte tiene sus propias leyes inherentes de desarrollo, y son estudiadas por un campo específico de investigación. El desarrollo social y económico afecta al desarrollo del arte de una forma importante. Pero no se pueden reducir de una forma mecánica. El desarrollo del arte, la literatura y la filosofía no refleja directamente la línea general de desarrollo de la sociedad y las fuerzas productivas. El ascenso y caída de las fuerzas productivas encuentra su expresión en la mente de hombres y mujeres de una forma contradictoria.

A fin de estudiar la conexión entre la producción cultural y la producción material es esencial, sobre todo, comprender a la última en su forma histórica determinada y no como una categoría 
general. Corresponde al modo de producción capitalista un tipo de producción cultural muy diferente a aquel que correspondía al modo de producción medieval ${ }^{9}$. La transformación del artista de productor de encargo en productor de mercancías se constituye en la respuesta lógica a las transformaciones que dieron lugar a su surgimiento.

En su obra The Invention of Art, el teórico norteamericano Larry Shiner expresa: «En el antiguo sistema del arte, el trabajo del productor era concreto en el sentido de que la facilidad, la inteligencia y la inventiva eran empleados para ejecutar un encargo que a menudo tenía un uso específico y un asunto que era acordado. En el sistema de mercado el trabajo se convierte en abstracto $^{10}$ en el sentido de que no está atado a un lugar o propósito específicos, no tiene un asunto predeterminado y, por tanto, tampoco tareas específicas de ejecución sino solo una generalizada creatividad $»^{11}$.

En este sentido se transita de una relación directa entre el productor de la obra de arte y su comprador, a una relación mediada por el mercado: el productor ya no es productor de la obra (trabajo concreto, encargo), ahora es productor de una obra mercancía (producida para el cambio), donde se genera y se realiza una nueva relación social. Bajo esta realidad, en un determinado momento histórico, aquel coincidente con el desarrollo y la consolidación del modo capitalista de producción, se podría considerar que el trabajo de los artistas habría empezado a ser tratado como cantidades intercambiables de tiempo de trabajo socialmente necesario bajo el mando de un capitalista.

\footnotetext{
9 Palma, L. M. y Aguado, L. F. «Economía de la Cultura. Una nueva área de especialización de la Economía». Revista de Economía Institucional, vol. 12, n. ${ }^{\circ}$ 22, primer semestre/2010, pp. 129-165. $10 \mathrm{El}$ autor asume que se refiere a la magnitud de valor, representada por el trabajo indiferenciado y al gasto de energías físicas y mentales. Esto es: el valor.

11 Shiner, L. (2001). "The invention of Art». Chicago: The University of Chicago Press, pp. $127-128$.
} 


\section{Algunas consideraciones sobre el contexto cubano}

En el plano de lo fenoménico de la sociedad cubana se manifiesta claramente la diferenciación de los productores culturales en cuanto a su diversidad, creatividad, elementos estéticos, históricos, entre otros. Si tomamos en consideración la comercialización del arte, se nos muestra como una esfera donde las relaciones monetario mercantiles tienen un papel clave, diferente, determinados por un nuevo diseño de la relación entre el artista y el consumidor, el cual ocupa un lugar mucho más importante en el sistema de valor, a partir de la utilización de las nuevas tecnologías de la información (TIC) que le da mayor autonomía al artista y donde se acorta el tiempo de realización del producto cultural convertido en mercancía.

El problema de las relaciones de propiedad y sus formas de expresión, materializada en los tipos socioeconómicos, tienen un lugar importante en el análisis de la relación cultura y economía en las condiciones de Cuba, sobre todo en lo referido al origen de los gastos, las formas de distribución de los medios de producción, y la retribución del sector de la cultura.

Sin embargo, a la luz de las condiciones actuales toma una gran importancia seguir profundizando en las formas y el lugar que ha adquirido el sector dentro del conjunto de la economía y comprender que la política económica tiene que reconocer el carácter especial de la producción artística de forma que se establezca una clara diferenciación metodológica, en cuanto a lo esencial y lo concreto, los procesos mediados por el mecanismo económico, la estructura de los intereses y los tipos socioeconómicos, los cuales determinan el proceso de producción cultural. 
La gestión de la actividad empresarial y de los recursos destinados a la actividad presupuestada del sector

Aplicar instrumentos científicos y metodológicos a la gestión de los procesos culturales en las organizaciones empresariales y presupuestadas, así como esfuerzos significativos en la capacitación de gestores y administradores.

Esta lógica de análisis debe tener presente las formas en que se manifiestan el sistema de contradicciones de la transición socialista, sobretodo la referida entre las formas de propiedad y los intereses que cada una determina, las cuales se reflejan en los aspectos generales y particulares de la producción cultural en las distintas manifestaciones, diferentes en estilo y contenido.

De esta forma, se puede llegar a comprender la complejidad en el análisis sobre la cuestión de la producción cultural en un contexto de una economía heterogénea, donde se transforma la realización de la propiedad social en la producción cultural y se manifiesta una relación dialéctica entre el aislamiento económico de los productores culturales por un lado y la producción socializada por otro, lo que condiciona la necesidad de ampliar el mecanismo de regulación económica al sector de la cultura.

Esta dinámica condiciona la necesidad objetiva de formas flexibles en la retribución del resultado final de la producción cultural en beneficio del productor, independientemente de la forma organizativa de la producción que haya elegido libremente, para lograr su realización efectiva y real, tomando en consideración lo estético y lo económicamente racional en función de los intereses individuales y sociales.

La antes mencionada estructura económica heterogénea, materializada en los diferentes tipos socioeconómicos que 
responden objetivamente a determinadas relaciones de propiedad, opera de forma contradictoria con las nuevas leyes económicas que surgen en este periodo, y complejizan su despliegue y cabal funcionamiento en todas las ramas y sectores de la economía y la sociedad en general, incluida la cultura y la producción de arte, donde se manifiesta de manera singular.

Esta lógica nos lleva a comprender el carácter contradictorio de la relación cultura y economía. De una parte, la interacción entre los procesos intrínsecos de la cultura, la tradición, el patrimonio, lo ético, lo estético, entre otros; y de otra parte, la posibilidad y necesidad objetiva de asumir la cultura como actividad económica generadora de recursos a través de su rentabilidad.

Desde esta óptica, el análisis toma un nuevo alcance desde el punto de vista de las relaciones socioeconómicas, que deben considerarse en el sentido del proceso de transformación de la cultura en mercancía a partir de definir la producción cultural como el proceso transformador de la creación artística en objeto de consumo para lograr su realización mercantil y socioeconómica.

El valor de la fuerza de trabajo se manifiesta como mercancía especial creadora de valor, donde se expresan en este caso aptitudes especiales que generan una relación indisoluble entre el creador y lo creado, (entre la idea y el hecho se interpone otro hecho) cuestión determinante en las relaciones sociales en este ámbito, en tanto el aislamiento del artista para crear es fuente de apropiación de lo creado, reconocido en el plano concreto como los derechos de autor.

La relación posesión de la fuerza de trabajo-posesión del resultado final en la producción artística en las condiciones de la transición al socialismo en Cuba, está objetivamente determinada por las nuevas trasformaciones socioeconómicas imperantes, 
tanto para el tipo de economía socialista, como para el tipo de economía de la pequeña producción mercantil, y sector privado, diferentes en esencia y en contenido.

Los ingresos como productor del artista, donde la fuerza de trabajo ha dejado de ser mercancía, en dependencia de la forma de apropiación del resultado final que él elija libremente, ha dejado de ser la expresión de la explotación y ha pasado a ser una categoría con un nuevo contenido cualitativo que expresa las relaciones de producción socialistas.

En las condiciones de Cuba, el artista productor se convierte objetivamente en productor socialista de mercancía cultural y sigue poseyendo la capacidad de crear un nuevo valor con la posibilidad de apropiarse del resultado de su trabajo, mediante los derechos de propiedad intelectual y derechos de autor.

La posición social de los artistas como productores de mercancías en un proceso de transición socialista se manifiesta en las relaciones de posesión y propiedad de su fuerza de trabajo así como de los productos de su trabajo, pero ahora con un nuevo contenido socioeconómico signado por la existencia de la propiedad social sobre los medios de producción.

En este escenario, la relación entre la producción cultural y el capital le va dando paso a una relación de causa efecto entre la existencia de la propiedad social sobre los medios de producción, materializada en la disponibilidad de recursos financieros y de capital a través del presupuesto del Estado, en forma de asignaciones y subvenciones; y la capacidad de creación en un ambiente desalienado, como artistas independientes o con algún tipo de relación contractual.

Este enfoque de la producción cultural está en correspondencia con la finalidad de esclarecer el sistema de leyes que regulan la formación económica social en Cuba. En esta, la cultura, los procesos culturales y la producción cultural se erigen como 
catalizadores de la presencia objetiva de tendencias y leyes económicas que expresan su naturaleza y contradicciones.

A diferencia de otras formaciones económicas sociales, lo que la producción artística revela entonces, es algo más sutil, porque a través de su trabajo consciente percibimos su relación ineludible con la sociedad en general y con las formas económicas presentes en la transición en particular, como elementos dinamizantes, en potencia, de la estructura de creación de valor en el nivel de la sociedad.

La producción artística acoge en su seno esta forma generalizada y jerarquizada de producir, en el movimiento del sujeto al objeto como propiedad materializada en este último, es decir como su valor. Es así que el valor en la producción cultural se manifiesta como un traspaso, en este caso del sujeto productor, capacitado como artista, al objeto producido, reconocido por la sociedad como obra de arte, para su pleno disfrute en un intento desalienante.

En las condiciones de Cuba se manifiesta una dicotomía: existe, por un lado, la garantía de la protección del Estado a toda la actividad cultural para garantizar un nivel cualitativamente superior en la renovación de los procesos identitarios y de satisfacción del ser humano, como fundamento de la justicia social y de la nación misma, en el marco actual de racionalidad económica; y por otro lado, la imperiosa e impostergable necesidad de situar al sector cultural en el lugar que le corresponde en cuanto a fuente indiscutida de crecimiento económico y de sector de avanzada en la gestión empresarial. 


\section{REFERENCIAS BIBLIOGRÁFICAS}

Achugar, H. (1999). «La incomprensible invisibilidad del ser económico, o acerca de cultura, valor y trabajo en América Latina». En García Canclini, N. y Moneta, C. (coords.). Las industrias culturales en la integración latinoamericana. Buenos Aires: Eudeba.

Adorno, T. y Horkheimer, M. (1988). «La industria cultural. Iluminismo como mistificación de masas». En Dialéctica del iluminismo. Buenos Aires: Editorial Sudamericana.

Álvarez Álvarez, L. y Barreto Argilagos, G. (2010). El arte de investigar el arte. Santiago de Cuba: Editorial Oriente.

Austin, T. (2000). «Para comprender el concepto de cultura». Revista UNAP Educación y Desarrollo, año 1, n. ${ }^{\circ}$ 1, Universidad Arturo Prat, Sede Victoria, IX Región de «La Araucanía», Chile.

Baumol, W. (1967). «Macroeconomics of Unbalanced Growth: The Anatomy of Urban Crisis». American Economic Review $57(3)$.

— (1967). «Performing Arts: The Permanent Crises». Business Horizons.

Bayardo, R. (1998). «Antropología, Identidad y Políticas Culturales». Programa de Antropología de la Cultura, Universidad de Buenos Aires. Disponible en: https://www.equiponaya. com.ar/articulos/identi01.htm.

BELL, D. (2006). Las contradicciones culturales del capitalismo. Madrid: Alianza Editorial.

Benhamou, F. (1997). La economía de la cultura. Montevideo: Ediciones Trilce.

Bernal, R. (9 de mayo de 2012). Entrevista concedida al autor por el Ministro de Cultura.

Bonet Agustí, L. (2001). Economía y cultura: Una reflexión en clave Latinoamericana. Barcelona: Oficina para Europa del BID. 
Carballo, J. A. (2012). «La relación Economía y Cultura». Disponible en: www.geocities.com/tomaustin_cl.

- (2012). «Hacia una revisión crítica de la Economía de la Cultura. Parte I». Revista Cuba Socialista. Disponible en: http://www.cubasocialista.cu/index.php?q=hacia-una-revision-critica-de-la-Economia-de-la-Cultura-parte-1.

- (2012): «Hacia una revisión crítica de la Economía de la Cultura. Parte II». Revista Cuba Socialista. Disponible en: http://www.cubasocialista.cu/index.php?q=hacia-una-revision-critica-de-la-Economia-de-la-Cultura-parte-2.

Castro Díaz-Balart, F. (2001). Ciencia, innovación y futuro. La Habana: Ediciones Especiales. Instituto Cubano del Libro.

Castro Ruz, F. (2008). «Palabras a los intelectuales». En Documentos de la Revolución Cubana, 1961. La Habana: Editorial de Ciencias Sociales.

Colectivo de autores (1961). Ensayos de estética marxista leninista. Uruguay: Ediciones Pueblos Unidos.

Colectivo de autores (2004). La economía política marxista. Reflexiones para un debate. La Habana: Editorial Félix Varela.

Cultura en Cifras Cuba (2010). Cuba: Oficina Nacional de Estadística.

Dantas, M. (2012). Nueva etapa de la industria cultural: de la lógica fabril a los «jardines amurallados». Buenos Aires: Ediciones Herramienta.

De Marchi, N. y Greene, J. (2005). «Adam Smith and Private Provision of the Arts». History of Political Economy, 37.

DiMaggio, P. y Useem, M. (1978). «Social Class and Arts Consumption: The Origins and Consequences of Class Differences in Exposure to the Arts in America». Theory and Society 5(2). Durán, J. M. (2006). «Arte y Capital. Releyendo el Capítulo VI de El Capital». Nómadas: Revista Crítica de Ciencias Sociales y Jurídicas. 
— (2011). «Elementos para una Crítica de la Economía Política del Arte». Revista de Economía Política de las Tecnologías de la Información y la Comunicación, vol. XIII, n. ${ }^{\circ} 2$.

Fernández Estrada, O. (2011). «El modelo de funcionamiento económico en Cuba y sus transformaciones. Seis ejes articuladores para un análisis». Revista Observatorio de la Economía y la Sociedad Latinoamericana, n. 154 . Disponible en: http://www. eumed.net/cursecon/ecolat/cu.

FERnÁNDEZ NúÑEZ, J. (1994). El mecanismo económico del socialismo. UH.

Fischer, S., Dornbusch, R. y Schmalensee, R. (1989). Economía (2. ${ }^{a}$ ed.). Madrid: Mcgraw-Hill/Interamericana de España.

Frey, B. (2000). Art and Economics. Heidelberg: Springer-Verlag (trad. esp.: La economía del arte. Barcelona: La Caixa.

GonzÁlez Arencibia, M. (2006). Globalización Cultural: Sus interacciones socioeconómicas y políticas. Retos para el desarrollo social. Eumed.net.

García CANCLINI, N. (1996). Culturas híbridas: estrategias para entrary salir de la modernidad. México D. F.: Editorial Grijalbo.

- (1997). Ideología, cultura y poder. Buenos Aires: Eudeba.

García Lorenzo, T. (2009). «Reflexiones a propósito de la economía de la cultura». Perfiles de la Cultura Cubana, mayo-diciembre.

Gramsci, A. (1916). «Socialismo y Cultura», Il Grido di Popolo (artículo reproducido en Ontología, Madrid: Sacristán de Siglo XXI).

KEYNES, J. M. (1981). La teoría general de la ocupación, el interés y el dinero (1. ${ }^{\mathrm{a}}$ ed. 1936). México: FCE.

Lenin, V. I. (1974). La literatura y el arte. La Habana: Editorial Arte y Sociedad. Instituto Cubano del Libro.

Lineamientos de la Política Económica y Social de la Revolución (2010). La Habana: Editora Política. 
LoucA, F. (s./f.). «Cultura, valores y ciclos largos del desarrollo capitalista». ISEG (Instituto Superior de Economia e Gestão), Universidade Técnica de Lisboa, UECE.

Luengo Cruz, M. (2008). «El producto cultural: claves epistemológicas de su estudio». Revista ZER, vol.13, n. ${ }^{\circ} 24$.

Marshall, A. (1954). Principios de economía (1. a ed. 1890). Madrid: Aguilar.

MarX, C. (1977). Manuscritos económicos y filosóficos de 1844. Editorial Pueblo y Educación.

(1978). Historia critica de la teoría de la plusvalía. Tomos I y II. La Habana: Editorial de Ciencias Sociales.

- (1982). El Capital. Tomo 1 (1. a ed. 1867). La Habana: Editora Política.

— (1982). Contribución a la Crítica de la Economía Política. La Habana: Editorial de Ciencias Sociales.

— (1989). Critica al Programa de Gotha. La Habana: Editorial de Ciencias Sociales.

Palma, L. (2004). «Mercado del arte y economía de la cultura». Cuadernos de Economía de la Cultura, 3.

- (2010). «Economía de la Cultura. Una nueva área de especialización de la Economía». Revista de Economía Institucional, vol. 12 , n. ${ }^{\circ} 22$.

Rifkin, J. (2000). La era del acceso. Barcelona: Editorial Paidós.

RoBins, L. (1984). Ensayo sobre la naturaleza y significación de la ciencia económica. Madrid: Editorial Prentice-Hall.

Rodríguez, J. L. (1995). «Cultura y Desarrollo». Conferencia inaugural presentada en el Primer Encuentro Iberoamericano sobre Cultura y Desarrollo. La Habana, del 19 al 23 de Noviembre de 1995.

Rubin, I. (2012). «Valor, precio de producción y trabajo productivo». En Ensayos sobre la teoría marxista del valor «Seminario de Lectura Metodológica de El Capital», pp. 32-51. 
SÁnchez NodA, R. (2009). La nueva Economía y el conocimiento: entre el mito y la realidad. La Habana: Editorial Félix Varela.

Throsby, D. (1999). «El papel de la música en el comercio internacional y en el desarrollo económico». En Informe Mundial sobre la Cultura, Unesco.

— (2002). Economía y Cultura. Madrid: Ediciones Akal.

Torres López, J. (1993). El estado crítico de la cultura. Madrid: Edit. FIM.

Towse, R. (1997). Cultural Economics: The Arts, the Heritage and the Media Industries, 2 vols. Cheltenham: Edward Elgar Publishing.

Williams, R. (1980). «Teoría cultural». En Marxismo y literatura. Barcelona: Península.

Woods, A. (2010). «El marxismo y el arte. Introducción a los escritos de Trotsky sobre arte». Disponible en: http://www. fundacionfedericoengels.org.

ZALLO, R. (1992). El mercado de la cultura: estructura económica y politica de la comunicación. San Sebastián: Grakoa. 